\title{
Atrophic Corpus Callosum Associated with Altered Functional Asymmetry in Major Depressive Disorder
}

This article was published in the following Dove Press journal: Neuropsychiatric Disease and Treatment

\author{
Shuhua Ran',* \\ Zhiwei Zuo ${ }^{2, *}$ \\ Chang $\mathrm{Li}^{3}$ \\ Xuntao Yin $\mathbb{1 D}^{4}$ \\ Wei $\mathrm{Qu}^{5}$ \\ Qianying Tang ${ }^{5}$ \\ Yao Wang (D' \\ Yanshu Shi' \\ Haitao $\mathrm{Li}^{\prime}$ \\ 'Department of Radiology, Southwest \\ Hospital, Army Medical University, \\ Chongqing, People's Republic of China; \\ ${ }^{2}$ Department of Radiology, General \\ Hospital of Western Theater Command, \\ Chengdu, People's Republic of China; \\ ${ }^{3}$ Department of Radiology, Daping \\ Hospital, Army Medical University, \\ Chongqing, People's Republic of China; \\ ${ }^{4}$ Department of Medical Imaging, \\ Guizhou Provincial People's Hospital, \\ Guizhou, People's Republic of China; \\ ${ }^{5}$ Department of Psychology, Southwest \\ Hospital, Army Medical University, \\ Chongqing, People's Republic of China \\ *These authors contributed equally to \\ this work
}

\begin{abstract}
Purpose: Impairments in intra- and inter-hemispheric information transfer circuits have been reported in patients with major depressive disorder (MDD). However, the specific anomalous connection (intra- and/or inter-hemispheric) and hemisphere (left and/or right) in which this connection plays a more dominant role in the pathogenic mechanism underlying MDD are still poorly understood.

Patients and Methods: Structural magnetic resonance imaging and resting-state functional magnetic resonance imaging were performed in 33 patients with MDD and 33 healthy controls. The intra- and inter-hemispheric functional connectivity (FC) strength in the default mode network areas and volume of the callosal subregions were computed using independent samples $t$-tests. The partial correlations between the volumes and FCs were also computed. Results: The patients with MDD had smaller volumes in the genu of the corpus callosum than the controls. The intrahemispheric FCs of the bilateral posterior cingulate gyrus, left precuneus, left medial superior frontal gyrus, left medial orbitofrontal gyrus, left angular gyrus and left middle temporal gyrus, and interhemispheric FCs of the bilateral posterior cingulate gyrus in the patients with MDD were lower than those in the controls. Moreover, the intrahemispheric FCs of the precuneus and interhemispheric FCs of middle frontal gyrus, orbital middle frontal gyrus, and anterior cingulate gyrus in the patients with MDD showed right-lateralized asymmetry, which were opposite from the asymmetry patterns observed in the controls. The functional asymmetry of the anterior cingulate gyrus was correlated with the volume of the genu of the corpus callosum and disease duration.

Conclusion: These findings provide robust evidence that intra- and inter-hemispheric disconnections are involved in MDD, and that functional disruptions in the left hemisphere may be more relevant to the pathophysiology of MDD. Furthermore, imbalanced interhemispheric exchanges may contribute to the anatomical deficits in the corpus callosum in patients with MDD.
\end{abstract}

Keywords: major depressive disorder, asymmetry, functional connectivity, corpus callosum

\section{Introduction}

Structural and functional asymmetry are fundamental biological traits in the human brain that may be attributed to lateral genetic expression and/or environmental influence. Each cerebral hemisphere has its own distinct strategy for multifarious perceptual and cognitive processing, such as handedness, language, and visuospatial functions. ${ }^{1}$ Moreover, emotional modulation is also lateralized within the cerebral hemispheres. Several models, such as the valence hypothesis and the
Correspondence: Haitao Li Email liihait@I63.com 
right hemisphere hypothesis, ${ }^{2,3}$ have been proposed to describe the relationship between emotions and cerebral lateralization, and some researchers believe that the right hemisphere may be more responsible for affective processing, while the left hemisphere may be more dominant for cognitive processing. ${ }^{2,3}$ The specialized functions within the bilateral hemispheres may be conducive to efficient computation and information communication.

Disrupted hemispheric asymmetry occurs in various neuropsychiatric diseases, such as social anxiety disorder, ${ }^{4}$ schizophrenia, ${ }^{5}$ epilepsy ${ }^{6}$ and major depressive disorder (MDD). ${ }^{7-10}$ As a severe mental disease, MDD involves emotional dysregulation that typically presents as excessive sadness, anhedonia and suicidality. Electroencephalography (EEG) studies have consistently verified a right-lateralized activated pattern of frontal alpha band signal in patients with MDD. $^{7-9}$ Our previous work ${ }^{10}$ demonstrated that the altered structural asymmetry in patients with MDD was mainly involved in cortical-striatal-pallidal-thalamic circuits. Notably, many resting-state functional MRI (rs-fMRI) studies have focused on the functional connectivity (FC) of the left and right cerebral hemispheres and consistently showed decreased interhemispheric $\mathrm{FCs}\left(\mathrm{FC}_{\text {inter }}\right)$ in $\mathrm{MDD} .{ }^{11-13}$ Furthermore, emerging evidence from task-based functional research suggests that the key pathogenic mechanisms underlying MDD may be deficient top-down (cortical-to-limbic) cognitive control and/or enhanced bottom-up (limbic-tocortical) emotional processing; ${ }^{14-16}$ however, these disrupted intrahemispheric communications in MDD, which can be represented as intrahemispheric $\mathrm{FCs}\left(\mathrm{FC}_{\text {intra }}\right)$ using rs-fMRI techniques, have not been adequately investigated. Whether the biased functional asymmetries were outcomes of $\mathrm{FC}_{\text {inter }}$ and/or $\mathrm{FC}_{\text {intra }}$ in patients with MDD remains unknown.

The corpus callosum is the largest of the intra- and inter-hemispheric myelinated axonal tracts that connect and integrate neural activity in the human brain. This formation of precise connections is essential for many aspects of higher neural functioning, including the integration of complex sensory information and regulation of cognitive and affective functions such as attention, memory, language, and abstract reasoning. ${ }^{17,18}$ As a physical pathway for information transfer within/between the hemispheres, the corpus callosum plays an important role in the balance of hemispheric activity. Research on split-brain patients has resulted in numerous insights into cerebral asymmetry and the interhemispheric exchange of information. ${ }^{19}$ For example, language is one of the most lateralized brain functions, with the left hemisphere being dominant in most people. Linguistic stimuli transmitted to the right hemisphere cannot be diverted to the left hemisphere in patients who undergo corpus callosotomy, and language-related areas located in the right hemisphere cannot process these stimuli properly, giving rise to lingual abnormalities. ${ }^{19,20}$ A thinning corpus callosum has been found in patients with bipolar disorder, ${ }^{21}$ and disrupted information exchange caused by impaired callosal function may lead to mood dysregulation in patients with bipolar disorder. $^{20}$ Bipolar disorder is a mood disorder characterized by mania and hypomania, and depression is its most common clinical feature. Moreover, impaired white matter integrity $^{22-24}$ and reduced volumes ${ }^{25-28}$ in the corpus callosum have been consistently observed in MDD in previous structural studies.

In the current study, we hypothesized that structural impairments in the corpus callosum may be associated with altered functional asymmetry in patients with MDD. Structural magnetic resonance imaging (MRI) and rs-fMRI were implemented to examine the callosal volumetric changes, asymmetric patterns of functional connectivity within default mode network (DMN) areas and the relationships between potential callosal volumetric changes and functional asymmetry in patients with MDD.

\section{Patients and Methods Subjects}

Thirty-three middle-aged patients diagnosed with MDD (21 females and 12 males) were included from the outpatient clinic of the Department of Psychology of our hospital. In an interview presided over by 2 experienced psychologists, the life-chart methodology was used to evaluate depression duration and severity using the 24item Hamilton Depression Scale $\left(\mathrm{HAM}-\mathrm{D}_{24}\right)$. The inclusion criteria were as follows: 1) patients aged between 18 and 48 years, 2) patients who met the Diagnostic and Statistical Manual of Mental Disorders IV (DSM-IV) diagnostic criteria for MDD, 3) patients who were newly diagnosed with a depressive episode and were not currently taking antidepressants, and 4) patients with moderate to severe depression (a total HAM-D 24 score $>20$ ). Thirty-three age-, sex- and education-matched healthy controls (HCs) who also received independent evaluations using the HAM-D ${ }_{24}$ were recruited for the study.

All subjects were right-handed, with no history of psychiatric or neurological disorders such as schizophrenia, bipolar disorder, Parkinson's disease, dementia, 
stroke, multiple sclerosis, brain tumors, or major medical diseases such as diabetes mellitus, heart failure, and anemia.

All subjects engaged in the study provided written informed consent before the study started. The Medical Ethics Committee of Southwest Hospital approved the study protocol and this study was conducted in accordance with the Declaration of Helsinki.

\section{MRI Acquisition}

Imaging data were obtained using a Siemens 3.0-Tesla Trio Tim MRI scanner (Siemens AG, Erlangen, Germany) equipped with a standard 12-channel phase-array head coil. To reduce head movement, sponge pads were placed on both sides of each participant's head. High-resolution structural T1weighted MR images and rs-fMRI scans were conducted on all participants. The structural T1WI used magnetizationprepared rapid gradient echo (MPRAGE) with the following parameters: $\mathrm{TR}=1900 \mathrm{~ms}, \mathrm{TE}=2.52 \mathrm{~ms}, \mathrm{FA}=9^{\circ}, \mathrm{FOV}=256$ $\times 256 \mathrm{~mm}^{2}$, slice thickness $=1 \mathrm{~mm}$, number of slices $=176$, and voxel size $=1 \times 1 \times 1 \mathrm{~mm}^{3}$. The resting functional scans were acquired using a 240 contiguous echo-planar imaging (EPI) sequence with the following settings: $\mathrm{TR}=2000 \mathrm{~ms}$, $\mathrm{TE}=30 \mathrm{~ms}, \mathrm{FA}=90^{\circ}$, slice thickness $=3 \mathrm{~mm}$, number of slices $=36, \mathrm{FOV}=192 \times 192 \mathrm{~mm}^{2}$, matrix size $=64 \times 64$ and voxel size $=3 \times 3 \times 3 \mathrm{~mm}^{3}$. All subjects were instructed before the scan to simply rest with their eyes closed and to not think of anything in particular or fall asleep.

\section{MRI Analysis}

Before analyzing the structural images, we examined whether the images were impacted by head movement. The qualified structural images were further volume segmented using FreeSurfer software (Massachusetts General Hospital, Boston, MA, USA, http://surfer.nmr.mgh.har vard.edu). The automated post-processing streams have been depicted in detail in previous studies ${ }^{29,30}$ and generally are as follows: Talairach coordinate system transform, bias-field correction, signal strength standardization, skull and soft tissues strip, volume labeling and white matter segmentation. The corpus callosum, including the genu (GCC), anterior midbody (aMCC), posterior midbody (pMCC), isthmus (ICC) and splenium (SCC) (Figure 1), was segmented based on a previously proposed classification. $^{31}$ The volumes of these 5 callosal subregions were then extracted.

The functional images were processed using the toolbox for Data Processing \& Analysis for Brain Imaging

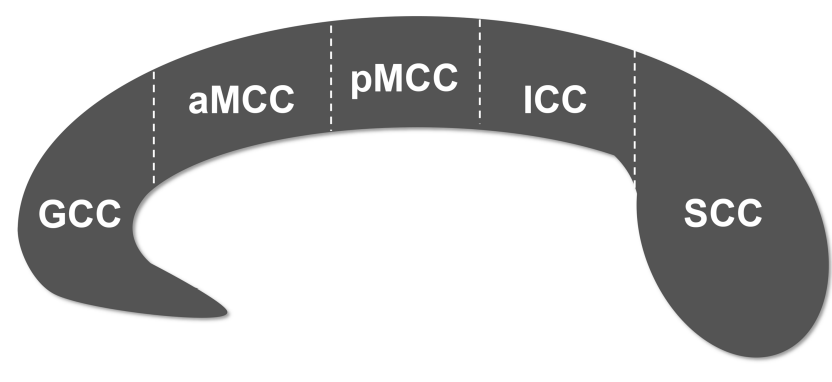

Figure I Subdivision of the corpus callosum into five subregions. Abbreviations: GCC, genu of corpus callosum; aMCC, anterior midbody of corpus callosum; pMCC, posterior midbody of corpus callosum; ICC, isthmus of corpus callosum; SCC, splenium of corpus callosum.

software (DPABI, version 3.0, http://www.rfmri.org/ dpabi), which is based on the Statistical Parametric Mapping (SPM) program (Wellcome Department of Cognitive Neurology, University College, London, UK, http://www.fil.ion.ucl.ac.uk/spm/software/spm12/) and the Resting-State fMRI Data Analysis Toolkit (REST, http:// www.restfmri.net), implemented in MATLAB R2012a (The MathWorks Inc., Natick, MA, USA). The details of the preprocessing procedures have been described in previous studies $5,11,32$ and primarily consist of the following steps. The first 10 volumes were discarded to stabilize the signal. The remaining 230 volumes were corrected for acquisition time differences between slices and for motion effects, and subjects with more than a $2 \mathrm{~mm}$ translation and $2^{\circ}$ maximal rotation were removed. The resulting data were spatially normalized to the Montreal Neurological Institute (MNI) space and were spatially smoothed with a 6-mm full-width Gaussian kernel at half maximum. A temporal filter $(0.01-0.08 \mathrm{~Hz})$ was applied to reduce the effect of low-frequency drifts and high-frequency physiological noise. Linear trends were removed via linear regression analysis. Per previous reports, ${ }^{33,34} 28 \mathrm{DMN}$ areas, including the bilateral medial superior frontal gyrus (mSFG), medial orbitofrontal gyrus (mOFG), middle frontal gyrus (MFG), orbital middle frontal gyrus (oMFG), anterior cingulate gyrus (ACG), posterior cingulate gyrus (PCG), hippocampus (HP), parahippocampal gyrus (PHG), inferior parietal lobule (IPL), supramarginal gyrus (SMG), angular gyrus (AG), precuneus (PC), middle temporal gyrus (MTG), and middle temporal pole (MTP), were selected, and the correlation coefficients between these areas were calculated based on the region of interest (ROI)-wise correlation approach for each participant. The standard ROIs were defined based on the Automated Anatomical Labeling (AAL) template. ${ }^{32,35}$ The resultant correlation coefficients were transformed to Fisher's 
$z$ scores to improve distribution normality. ${ }^{36}$ The average coefficient (absolute value) of an ROI with other ROIs from the ipsilateral hemisphere was designated the ROI's $\mathrm{FC}_{\text {intra }}$, and that from the contralateral hemisphere was designated the ROI's $\mathrm{FC}_{\text {inter }}$. To quantify the $\mathrm{FC}$ differences between the left and right hemispheres, the asymmetry index (AI) was computed for each ROI using the following formula: ${ }^{10,32,37} \mathrm{AI}=($ left $\mathrm{FC}-$ right $\mathrm{FC}) \diamond 100 /$ (left FC + right FC). The AI values ranged from -100 (entirely rightward lateralization) to +100 (entirely leftward lateralization).

\section{Statistical Analysis}

Demographic characteristics and clinical features were compared between patients with MDD and the HCs using the independent sample $t$-test (age and HAM-D 24 score), MannWhitney $U$-test (education level) and chi-square test (sex). The independent two-sample $t$-test was used to assess significant differences in the volumes of the callosal subregions, $\mathrm{FC}_{\text {intra, }}$, $\mathrm{FC}_{\text {inter, }}$ and the $\mathrm{AIs}$ of $\mathrm{FC}_{\text {intra }}\left(\mathrm{AI}_{\text {intra }}\right)$ and $\mathrm{FC}_{\text {inter }}\left(\mathrm{AI}_{\text {inter }}\right)$ between the patients with MDD and the HCs. Partial correlation analysis was performed to explore the relationships between structural and functional parameters with statistically significant differences and the clinical features of patients with MDD. Age, sex, and education level were imported as covariates. Statistical analyses were performed using SPSS 22.0 software (IBM Inc., Armonk, New York, USA). The Bonferroni method or false discovery rate (FDR) correction was applied to the between-group analyses and correlation analyses that involved multiple comparisons.

\section{Results}

\section{Participants' Characteristics}

Table 1 displays the participants' demographic and clinical information. The MDD and HC groups were matched for age, sex, and education level $(p>0.05)$. The HAM- $\mathrm{D}_{24}$ scores of patients with MDD were significantly higher than those of the HCs $(p<0.05)$.

\section{Callosal Volume Analysis}

Compared with HC group, the MDD group showed significantly smaller GCC volumes (Bonferroni-corrected $p<$ 0.05). No significant differences were found in the aMCC, pMCC, ICC or SCC volumes between the MDD and HC groups (Bonferroni-corrected $p>0.05$ ) (Table 2) or in the total corpus callosum volumes between the MDD and HC groups $(p>0.05)$.
Table I Demographic Features

\begin{tabular}{|c|c|c|c|c|}
\hline Characteristics & $\begin{array}{l}\text { MDD } \\
(n=33)\end{array}$ & $\begin{array}{l}\mathrm{HC} \\
(n=33)\end{array}$ & $\begin{array}{l}\text { Diagnosis } \\
\text { Effect }\end{array}$ & $p$ \\
\hline Age, years & $28.76 \pm 1.58$ & $31.64 \pm 1.29$ & $t=-|.4|$ & 0.162 \\
\hline Sex, female:male & $21: 12$ & $18: 15$ & $\chi^{2}=0.56$ & 0.453 \\
\hline Education, years & $13.79 \pm 0.52$ & $13.67 \pm 0.69$ & $u=-0.18$ & 0.855 \\
\hline $\begin{array}{l}\text { Duration } \\
\text { of disorder, years }\end{array}$ & $1.60 \pm 0.26$ & - & - & - \\
\hline HAM-D 24 score & $30.88 \pm 0.69$ & $2.61 \pm 0.27$ & $t=38.24$ & $<0.0001$ \\
\hline
\end{tabular}

Notes: Continuous variables are expressed as mean \pm SEM. Statistical significance: $p$ value $<0.05$.

Abbreviations: MDD, major depressive disorder; HC, healthy control; HAM-D ${ }_{24}$, 24-item Hamilton Depression Scale.

Table 2 Volumes of Callosal Subregions in the MDD and HC Groups

\begin{tabular}{|l|l|l|l|l|}
\hline Subregions & \multicolumn{2}{|l|}{ Volume $\left(\mathrm{mm}^{\mathbf{3}}\right)$} & $\boldsymbol{t}$ & $\boldsymbol{P}$ \\
\hline & MDD & HC & & \\
\hline GCC & $821.60 \pm 23.20$ & $908.89 \pm 18.79$ & -2.92 & $0.005^{*}$ \\
aMCC & $537.64 \pm 22.99$ & $548.91 \pm 27.62$ & -0.31 & 0.755 \\
PMCC & $553.87 \pm 22.72$ & $542.01 \pm 26.02$ & 0.34 & 0.732 \\
ICC & $501.55 \pm 16.25$ & $480.01 \pm 20.70$ & 0.82 & 0.416 \\
SCC & $943.01 \pm 26.44$ & $974.58 \pm 24.53$ & -0.88 & 0.385 \\
\hline
\end{tabular}

Notes: Continuous variables are expressed as mean \pm SEM. *Bonferroni-corrected $p=0.05 / 5=0.01$.

Abbreviations: MDD, major depressive disorder; $\mathrm{HC}$, healthy control; GCC, genu of the corpus callosum; aMCC, anterior midbody of the corpus callosum; PMCC, posterior midbody of the corpus callosum; ICC, isthmus of the corpus callosum; SCC, splenium of the corpus callosum.

\section{Functional Connectivity Analysis}

The FC differences between the MDD and HC groups were visualized using the BrainNet Viewer (http://www.nitrc.org/ projects/bnv/) (Figure 2). Supplementary Table 1 shows the average FC strength for all ROIs. Compared with the HC group, the MDD group exhibited significantly lower $\mathrm{FC}_{\text {intra }}$ strength in the left PCG $(t=-4.62$, FDR-corrected $p=$ $0.0003)$, right PCG $(t=-3.29$, FDR-corrected $p=0.03)$, left mSFG $(t=-2.66$, FDR-corrected $p=0.03)$, left mOFG $(t=-3.69$, FDR-corrected $p=0.003)$, left AG $(t=-2.70$, FDR-corrected $p=0.03)$, left PC $(t=-2.95$, FDR-corrected $p=0.02)$ and left MTG $(t=-2.55$, FDR-corrected $p=0.03)$ (Figure 2A), and lower $\mathrm{FC}_{\text {inter }}$ strength in the left PCG $(t=$ -3.26, FDR-corrected $p=0.03)$ and right PCG $(t=-3.77$, FDR-corrected $p=0.005$ ) (Figure 2B). No significantly greater $\mathrm{FC}_{\text {intra }}$ or $\mathrm{FC}_{\text {inter }}$ strength was observed in the patients with MDD.

Compared with the HCs, the MDD group showed significantly lower $\mathrm{AI}_{\text {intra }}$ in the PC $(t=-3.33$, FDRcorrected $p=0.01)$ and lower $\mathrm{AI}_{\text {inter }}$ in the MFG $(t=$ 

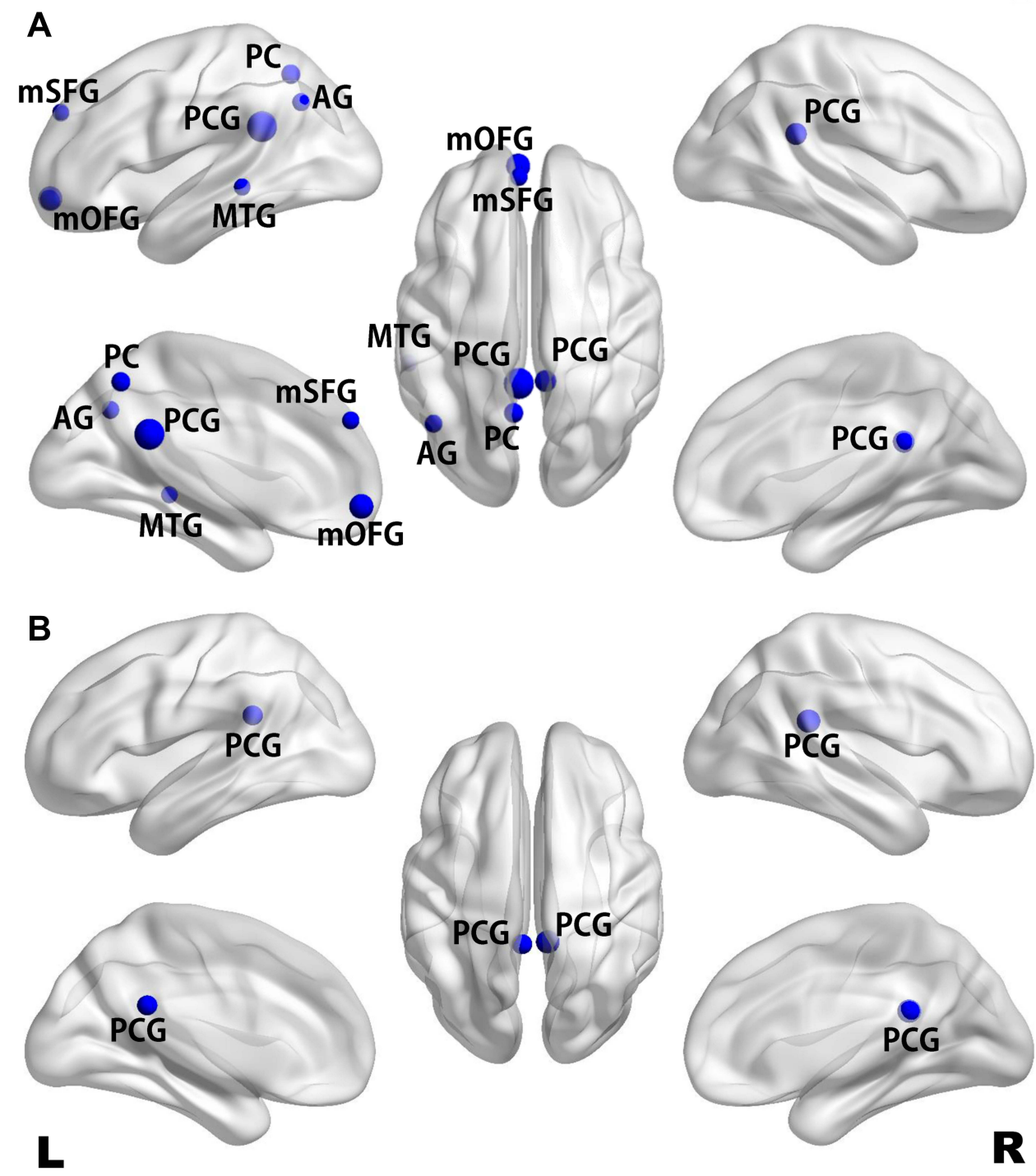

Figure 2 Maps of significant differences in intrahemispheric (A) and interhemispheric (B) functional connectivity between the MDD and HC groups. Differences in functional connectivity strength are presented on a standard translucent brain. Greater nodes indicate greater differences (FDR-corrected $p<0.05$ ).

Abbreviations: MDD, major depressive disorder; HC, healthy control; AG, angular gyrus; mOFG, medial orbitofrontal gyrus; mSFG, medial superior frontal gyrus; MTG, middle temporal gyrus; PC, precuneus; PCG, posterior cingulate gyrus.

-3.75$, FDR-corrected $p=0.005)$, oMFG $(t=-3.45$, FDRcorrected $p=0.007)$ and ACG $(t=-3.24$, FDR-corrected $p=0.009$ ) (Figure 3 ). No significantly greater $\mathrm{AI}_{\text {intra }}$ or $\mathrm{AI}_{\text {inter }}$ was observed in the patients with MDD.

\section{Correlation Analysis}

The partial correlation analysis revealed a significant positive correlation between the GCC volume and $\mathrm{AI}_{\text {inter }}$ of the ACG $(r=0.50, p=0.005)$, a negative correlation between the HAM-D ${ }_{24}$ scores and $\mathrm{AI}_{\text {inter }}$ of the mOFG $(r=-0.48$, $p=0.008)$, and a negative correlation between the disease duration and $\mathrm{AI}_{\text {inter }}$ of the ACG $(r=-0.47, p=0.009)$ in the patients with MDD (Figure 4).

\section{Discussion}

The DMN is strongly correlated with controlling and processing emotional/cognitive behaviors and self-generated thought, $^{38}$ and the PCG, mSFG, mOFG, AG and PC are the core hubs of the DMN, allowing information to communicate within the DMN subsystems, including the medial temporal subsystem and the dorsal medial subsystem. ${ }^{38}$ The $\mathrm{FC}_{\text {intra }}$ strength in the bilateral PCG, and left $\mathrm{mSFG}$, mOFG, AG, and PC was significantly decreased, and the left PCG showed the greatest differences between the groups (Figure 2A); only the PCG showed concurrent decreases in the $\mathrm{FC}_{\text {intra }}$ and $\mathrm{FC}_{\text {inter }}$ strength in both hemispheres (Figure 2), reflecting that functional 

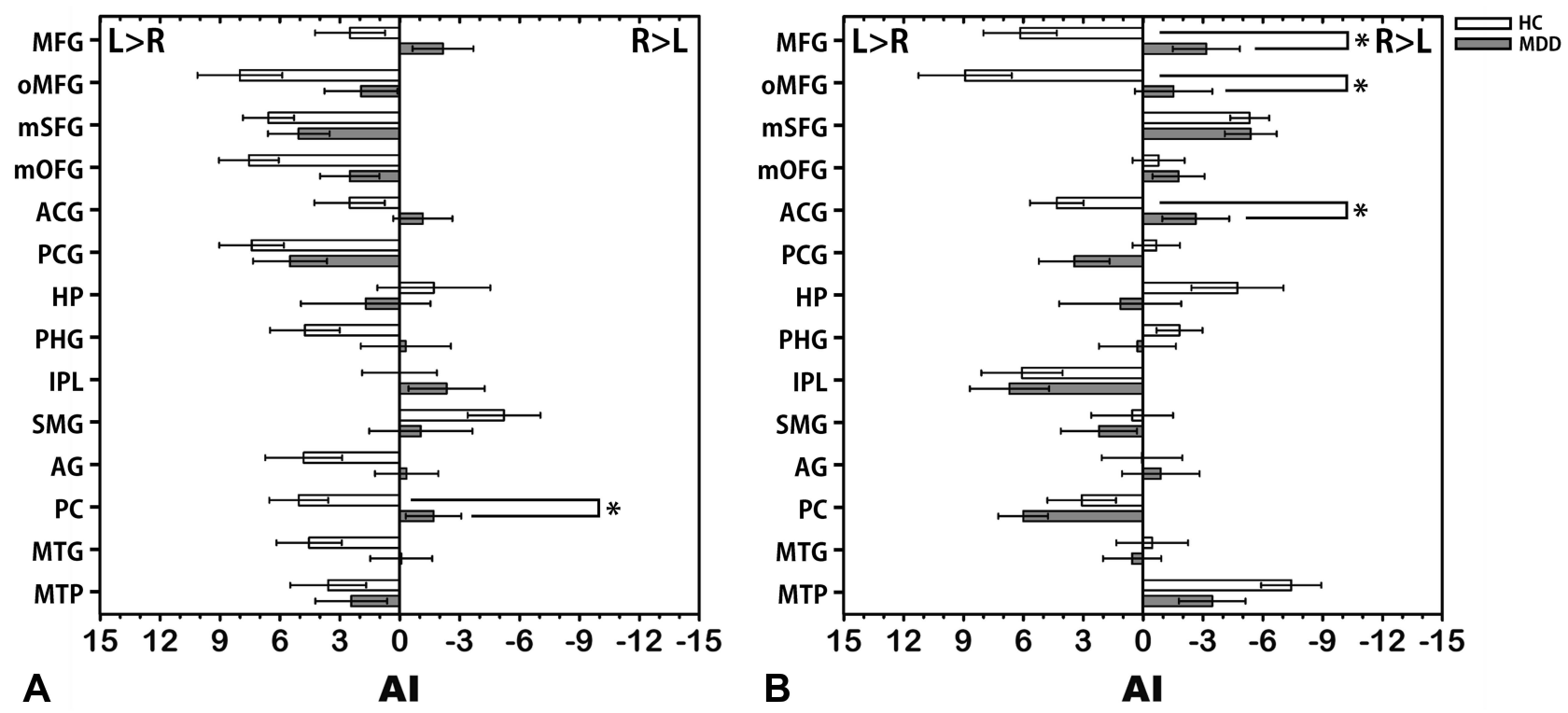

Figure 3 Mean Al of intrahemispheric (A) and interhemispheric (B) functional connectivity in the MDD and HC groups. The error bars represent standard errors. *Indicates significant differences between groups (FDR-corrected $p<0.05$ ).

Abbreviations: Al, asymmetry index; MDD, major depressive disorder; HC, healthy control; ACG, anterior cingulate gyrus; AG, angular gyrus; HP, hippocampus; IPL, inferior parietal lobule; MFG, middle frontal gyrus; mOFG, medial orbitofrontal gyrus; mSFG, medial superior frontal gyrus; MTG, middle temporal gyrus; MTP, middle temporal pole; oMFG, orbital part middle frontal gyrus; PC, precuneus; PCG, posterior cingulate gyrus; PHG, parahippocampal gyrus; SMG, supramarginal gyrus.
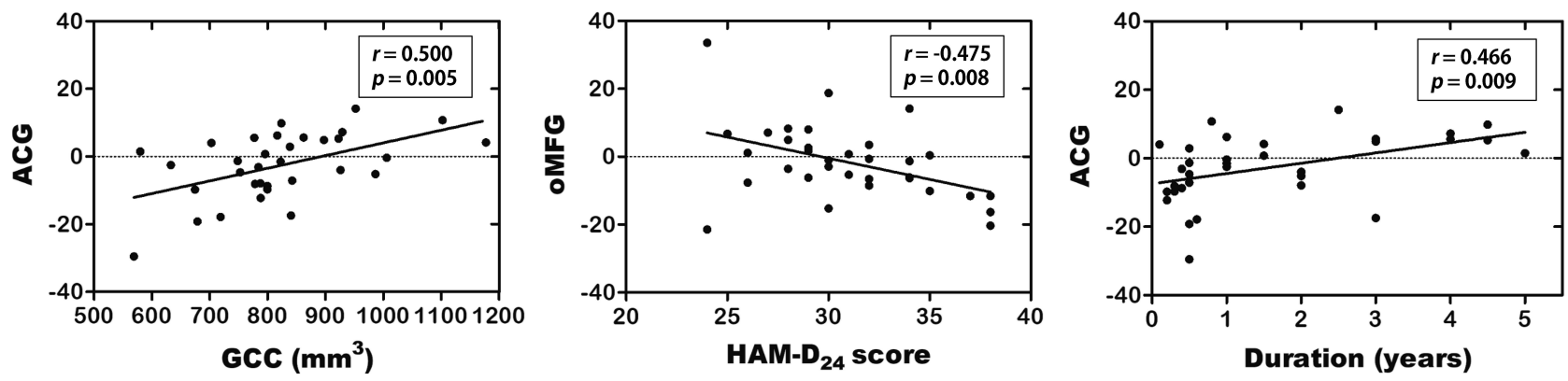

Figure 4 Correlations between $A C G \mathrm{Al}_{\text {inter }}$ and $\mathrm{GCC}$ volume, oMFG $\mathrm{Al}_{\text {inter }}$ and HAM- $\mathrm{D}_{24}$ score, and $A C G \mathrm{Al}$ inter and duration in patients with MDD. The partial correlation coefficients were corrected for age, sex and education level.

Abbreviations: $\mathrm{Al}_{\text {inten }}$ asymmetry index of interhemispheric functional connectivity strength; $\mathrm{ACG}$, anterior cingulate gyrus; GCC, genu of corpus callosum; oMFG, orbital part middle frontal gyrus; HAM-D 24 , 24-item Hamilton Depression Scale; MDD, major depressive disorder.

dysconnectivity of the PCG could be a prominent feature of MDD. The PCG plays an important role in internally directed cognition, attention focus, ${ }^{39}$ episode memory, ${ }^{38}$ and possibly control of responses prompting environmental changes. ${ }^{40}$ The PCG also has a strong connectivity with other intrinsic networks such as the frontoparietal control network and the dorsal attention network, ${ }^{39-42}$ which are involved in cognition processing. Local PCG impairments or dysregulated intrinsic networks may result in inappropriate functional activities in the PCG, which lead to a distraction of internally directed information processing in patients with MDD. The mSFG and mOFG are parts of the media prefrontal gyrus (mPFG), which controls higher- level cognition and socioemotional and executive behaviors ${ }^{43}$ and belongs to the frontoparietal control network. ${ }^{42}$ To maintain proper behaviors when adapting to specific circumstances, the mPFG is critical for coordinating sustained attention and flexibly restraining improper responses. ${ }^{44}$ However, acute or chronic stress can increase the risk of depression ${ }^{45}$ by initiating dysregulated processes involving the autonomic nervous system and hypothalamic-pituitary-adrenocortical (HPA) axis, ultimately resulting in damaged performances in spatial working memory, behavioral flexibility, decision-making and planning, which are the primary executive functions of the $\mathrm{mPFG}^{46} \mathrm{~A}$ task-based fMRI study found that $\mathrm{mPFG}$ 
and PCG activation is related to rumination during nonself-relevant tasks in patients with MDD, revealing the functional basis of persistent immersion in self-focus in patients with MDD. ${ }^{47}$

To our knowledge, this is the first study to explore asymmetric changes in FCs in patients with MDD. Interestingly, more brain areas showed changes in the $\mathrm{FC}_{\text {intra }}$ than $\mathrm{FC}_{\text {inter, }}$, while more brain areas showed changes in $\mathrm{AI}_{\text {inter }}$ than $\mathrm{AI}_{\text {intra }}$, suggesting that both intra- and interhemispheric activities were significantly disrupted. Although the MFG, oMFG and ACG in patients with MDD did not show significant changes in interhemispheric connectivity, their rightward asymmetric patterns were significantly reversed from those of the HCs. Therefore, examining alterations in the functional asymmetry of depression may provide subtle pathophysiological information and potential therapeutic targets. Decreases in the $\mathrm{PC}_{\mathrm{AI}_{\text {intra }}}$ and the $\mathrm{AI}_{\text {inter }}$ of the MFG, oMFG and ACG in patients with MDD occurred mainly because the average FC strength of the left side of those areas was lower than that of the right side, which was contrary to that of the HCs (Supplementary Table 1). Furthermore, with increased depressive severity (using HAM- ${ }_{24}$ ), the interhemispheric connectivity of the oMFG in patients with MDD was lateralized more to the right than the left. Various studies have demonstrated hypoperfusion in the left hemisphere and hyperperfusion in the right hemisphere in patients with MDD. ${ }^{48-50}$ Since the left hemisphere may be more efficient in cognitive control, and the right hemisphere may be more efficient in affective processing, ${ }^{2,3}$ dysfunction in the left hemisphere may cause deficits in sufficient cognitive control of emotional processing, while relatively greater functional activity in the right hemisphere may cause excessive emotional processing. However, our results show that decreased intrahemispheric connectivity and interhemispheric index within DMN areas in patients with MDD were concentrated in the left hemisphere, suggesting the possibility that MDD is more associated with functional abnormalities in left cerebral areas implicated in the cognitive modulation of affective states. Findings from a functional study on emotional judgment ${ }^{48}$ may support our speculation. These finding show that left prefrontal cortex activity is negatively associated with negative emotional valence and positively associated with positive emotional valence in patients with MDD. However, the controls displayed opposite relationships, and the right prefrontal cortex displayed no correlations with positive/negative emotional valence in patients with MDD, indicating a dissociation of the left and right prefrontal cortex depending on the emotional valence in the MDD. Moreover, unilateral damage to cerebral areas is more likely to contribute to depression, especially when the lesions are in the left hemisphere. ${ }^{51}$

In the current study, the GCC showed volumetric changes in patients with MDD, which is consistent with morphological studies involving adults with early-onset depression, ${ }^{25,27}$ adolescents with early-onset depression ${ }^{26}$ and elderly individuals with late-onset depression. ${ }^{28}$ Volumetric decreases in the anterior corpus callosum also occurred in etiology-based recombinant mouse models of depression, which were established by replacing a homologous mouse DNA sequence with a 6-base DNA sequence from the human CREB1 promoter, which is related to development of recurrent, early-onset MDD. ${ }^{52}$ However, our results differed from those of a previous study, ${ }^{53}$ which suggested that an increased corpus callosum volume may be confined to patients with familial MDD. Methodological heterogeneities in imaging features, morphometric techniques, patient samples, disease severity, and illness duration may lead to such inconsistencies. Furthermore, some participants in that study ${ }^{53}$ had a history of antidepressant use, and long-term therapies with different antidepressants can block or even reverse neuronal atrophy and cell loss by increasing neurotrophic factor expressions. ${ }^{54}$ The present findings of callosal atrophy restricted to the GCC may be related to the topographic arrangement of transcallosal fiber tracts. The defined boundaries of the corpus callosum are somewhat arbitrary because the corpus callosum has no morphologically macroscopic interior borders. Per Hofer and Frahm's new scheme of vertical corpus callosum segmentations, ${ }^{31}$ the GCC is the major white matter commissure connecting the prefrontal cortex; the aMCC and pMCC comprise projections connected with motor areas such as the premotor, supplementary motor and primary motor cortex; the SCC contains fibers projecting into somatosensory cortical regions; and the ICC contains fibers projecting into the auditory, visual, and tempo-parietal regions. Although structural and functional changes in the motor, sensory, auditory and visual-related cerebral cortex can also manifest in $\mathrm{MDD},{ }^{55,56}$ deficits in the prefrontal cortex are one major abnormality in patients with MDD. ${ }^{57,58}$ Otherwise, microstructural abnormalities in the GCC, which are related to the loss of white matter integrity, may be a common feature in depression. ${ }^{59-61}$ In addition, cerebral blood flow and glucose metabolism in depressed 
individuals are reduced in the GCC, ${ }^{62}$ suggesting that the GCC may play a more important role than other parts of the corpus callosum in MDD pathophysiology.

The GCC may also play an important role in functional asymmetry. Most fibers symmetrically interconnect the homotopic anatomical regions of the two hemispheres, but some heterotopic fibers also asymmetrically project to cortical regions with different functions. ${ }^{31}$ Moreover, depression has been linked to demyelination processes in the corpus callosum, ${ }^{63}$ which may lead to a reduced quantity and speed of information transfer between hemispheres, ${ }^{64}$ and antidepressants may function via specific signaling mechanisms, such as glycogen synthase kinase, which govern myelination to achieve therapeutic effects. ${ }^{65}$ The ACG is topologically located anterior to the GCC, and during corpus callosum development, allelic commissural projections from the ACG are the first axons to form the midline bundles of the corpus callosum. ${ }^{66}$ The ACG is especially crucial for affective processing and executive controls given its strong connections with prefrontal, limbic and paralimbic areas and is related to negative emotion-related behaviors such as depression, pain and stress. ${ }^{66}$ As the depressive duration increases, the interhemispheric connectivity of the ACG shows a more leftward asymmetry. Thus, we consider this result a compensatory mobilization for a long-term deficient functional ACG activity, which attempts to maintain affective function, since enhanced ACG activation is considered a promising indicator of therapeutic response in patients with MDD. ${ }^{67}$ However, the morphological changes observed in depression seem to be chronic alterations of dendrites, axons and synapses, ${ }^{68}$ whereas the positive correlation between duration and the functional asymmetry of the ACG suggests that the rightward asymmetry of the ACG had already occurred during the early stages in patients with MDD. Therefore, the positive relationship between the atrophic GCC and interhemispheric asymmetry of the ACG found in this study suggests that structural abnormalities in the corpus callosum might be ascribed to disturbed interhemispheric interactions in patients with MDD.

Several limitations of the present study should be acknowledged. First, this cross-sectional investigation had a relatively small sample size, thus the morphometric and functional findings may be noncategorical, and further longitudinal studies are necessary to detect potential variations in functional asymmetry following different durations of depression. Second, we segmented the corpus callosum into five sections based on cortical projections, and potential morphometric or functional alterations in other refined subregions may have been missed. Third, only DMN areas were selected for the FC analysis. Although these cerebral areas are strongly linked with MDD, other brain regions that may contribute to MDD pathophysiology were unexamined in this study, and these regions may also exist in altered FC asymmetry. In addition, further studies may be needed to explore the relationship between the white matter integrity of the corpus callosum and functional asymmetry in MDD.

\section{Conclusion}

Reduced information exchanges were found within intrahemispheric and between interhemispheric DMN in middle-aged patients with MDD, and the dysfunction levels of intra- and inter-hemispheric communications were comparable. This study provides important new evidence for the potential contributions of altered functional asymmetry to MDD, and suggests that functional asymmetry can be a promising biomarker of condition assessment and even treatment surveillance for MDD. Particularly, ACG asymmetry was related to volumetric decreases in the GCC and to disease duration, indicating that aberrant interhemispheric connections may contribute to the anatomical deficits in the corpus callosum in patients with MDD.

\section{Acknowledgments}

This study was funded by the National Key Research and Development Plan of China (grant No. 2016YFC0107101) and the National Nature Science Foundation of China (grant No. 81471647).

\section{Disclosure}

The authors report no conflicts of interest in this work.

\section{References}

1. Kong XZ, Mathias SR, Guadalupe T, et al. Mapping cortical brain asymmetry in 17,141 healthy individuals worldwide via the ENIGMA consortium. Proc Natl Acad Sci U S A. 2018;115(22):E5154-E5163. doi:10.1073/pnas.1718418115

2. Gainotti G. Unconscious processing of emotions and the right hemisphere. Neuropsychologia. 2012;50(2):205-218. doi:10.1016/j. neuropsychologia.2011.12.005

3. Wittling W, Roschmann R. Emotion-related hemisphere asymmetry: subjective emotional responses to laterally presented films. Cortex. 1993;29(3):431-448. doi:10.1016/S0010-9452(13)80252-3

4. Jung YH, Shin JE, Lee YI, Jang JH, Jo HJ, Choi SH. Altered amygdala resting-state functional connectivity and hemispheric asymmetry in patients with social anxiety disorder. Front Psychiatry. 2018;9:164. doi:10.3389/fpsyt.2018.00164

5. Zhu F, Liu F, Guo W, et al. Disrupted asymmetry of inter- and intra-hemispheric functional connectivity in patients with drug-naive, first-episode schizophrenia and their unaffected siblings. EBioMedicine. 2018;36:429-435. doi:10.1016/j.ebiom.2018.09.012 
6. Shah P, Bassett DS, Wisse LEM, et al. Structural and functional asymmetry of medial temporal subregions in unilateral temporal lobe epilepsy: a 7T MRI study. Hum Brain Mapp. 2019;40 (8):2390-2398. doi:10.1002/hbm.24530

7. Chang JS, Yoo CS, Yi SH, et al. An integrative assessment of the psychophysiologic alterations in young women with recurrent major depressive disorder. Psychosom Med. 2012;74(5):495-500. doi:10.1097/PSY.0b013e31824d0da0

8. Schaffer CE, Davidson RJ, Saron C. Frontal and parietal electroencephalogram asymmetry in depressed and nondepressed subjects. Biol Psychiatry. 1983;18(7):753-762.

9. Zotev V, Yuan H, Misaki M, et al. Correlation between amygdala BOLD activity and frontal EEG asymmetry during real-time fMRI neurofeedback training in patients with depression. Neuroimage Clin. 2016;11:224-238. doi:10.1016/j.nicl.2016.02.003

10. Zuo Z, Ran S, Wang Y, et al. Asymmetry in cortical thickness and subcortical volume in treatment-naive major depressive disorder. Neuroimage Clin. 2019;21:101614. doi:10.1016/j.nicl.2018.101614

11. Guo W, Liu F, Dai Y, et al. Decreased interhemispheric resting-state functional connectivity in first-episode, drug-naive major depressive disorder. Prog Neuropsychopharmacol Biol Psychiatry. 2013;41:24-29. doi:10.1016/j.pnpbp.2012.11.003

12. Yang $\mathrm{H}$, Wang $\mathrm{C}$, Ji G, et al. Aberrant interhemispheric functional connectivity in first-episode, drug-naive major depressive disorder. Brain Imaging Behav. 2019;13(5):1302-1310. doi:10.1007/s11682018-9917-x

13. Wang L, Li K, Zhang QE, et al. Interhemispheric functional connectivity and its relationships with clinical characteristics in major depressive disorder: a resting state fMRI study. PLoS One. 2013;8 (3):e60191. doi:10.1371/journal.pone.0060191

14. Fales CL, Barch DM, Rundle MM, et al. Altered emotional interference processing in affective and cognitive-control brain circuitry in major depression. Biol Psychiatry. 2008;63(4):377-384. doi:10.1016/j.biopsych.2007.06.012

15. Strigo IA, Matthews SC, Simmons AN. Decreased frontal regulation during pain anticipation in unmedicated subjects with major depressive disorder. Transl Psychiatry. 2013;3(3):e239. doi:10.1038/tp.2013.15

16. Mayberg HS, Liotti M, Brannan SK, et al. Reciprocal limbic-cortical function and negative mood: converging PET findings in depression and normal sadness. Am $J$ Psychiatry. 1999;156(5):675-682. doi:10.1176/ajp.156.5.675

17. Edwards TJ, Sherr EH, Barkovich AJ, Richards LJ. Clinical, genetic and imaging findings identify new causes for corpus callosum development syndromes. Brain. 2014;137(Pt 6):1579-1613. doi:10.1093/ brain/awt358

18. Fenlon LR, Richards LJ. Contralateral targeting of the corpus callosum in normal and pathological brain function. Trends Neurosci. 2015;38(5):264-272. doi:10.1016/j.tins.2015.02.007

19. Gazzaniga MS. Cerebral specialization and interhemispheric communication: does the corpus callosum enable the human condition? Brain. 2000;123(Pt 7):1293-1326. doi:10.1093/brain/123.7.1293

20. van der Knaap LJ, van der Ham IJ. How does the corpus callosum mediate interhemispheric transfer? A review. Behav Brain Res. 2011;223(1):211-221. doi:10.1016/j.bbr.2011.04.018

21. Walterfang M, Malhi GS, Wood AG, et al. Corpus callosum size and shape in established bipolar affective disorder. Aust $N$ Z J Psychiatry. 2009;43(9):838-845. doi:10.1080/00048670903107534

22. Liao $\mathrm{Y}$, Huang $\mathrm{X}, \mathrm{Wu} \mathrm{Q}$, et al. Is depression a disconnection syndrome? Meta-analysis of diffusion tensor imaging studies in patients with MDD. J Psychiatry Neurosci. 2013;38(1):49-56. doi:10.1503/ jpn. 110180

23. Korgaonkar MS, Grieve SM, Koslow SH, Gabrieli JD, Gordon E, Williams LM. Loss of white matter integrity in major depressive disorder: evidence using tract-based spatial statistical analysis of diffusion tensor imaging. Hum Brain Mapp. 2011;32 (12):2161-2171. doi:10.1002/hbm.21178
24. Chen G, Hu X, Li L, et al. Disorganization of white matter architecture in major depressive disorder: a meta-analysis of diffusion tensor imaging with tract-based spatial statistics. Sci Rep. 2016;6:21825. doi:10.1038/srep21825

25. Kemp A, MacMaster FP, Jaworska N, et al. Age of onset and corpus callosal morphology in major depression. J Affect Disord. 2013;150 (2):703-706. doi:10.1016/j.jad.2013.05.009

26. Macmaster FP, Carrey N, Marie Langevin L. Corpus callosal morphology in early onset adolescent depression. J Affect Disord. 2013;145(2):256-259. doi:10.1016/j.jad.2012.04.047

27. Lyoo IK, Kwon JS, Lee SJ, et al. Decrease in genu of the corpus callosum in medication-naive, early-onset dysthymia and depressive personality disorder. Biol Psychiatry. 2002;52(12):1134-1143. doi:10.1016/S0006-3223(02)01436-1

28. Ballmaier M, Kumar A, Elderkin-Thompson V, et al. Mapping callosal morphology in early- and late-onset elderly depression: an index of distinct changes in cortical connectivity. Neuropsychopharmacology. 2008;33(7):1528-1536. doi:10.1038/sj. npp. 1301538

29. Dale AM, Fischl B, Sereno MI. Cortical surface-based analysis. I. Segmentation and surface reconstruction. Neuroimage. 1999;9 (2):179. doi:10.1006/nimg.1998.0395

30. Fischl B, Dale AM. Measuring the thickness of the human cerebral cortex from magnetic resonance images. Proc Natl Acad Sci U S A. 2000;97(20):11050-11055. doi:10.1073/pnas.200033797

31. Hofer S, Frahm J. Topography of the human corpus callosum revisited-comprehensive fiber tractography using diffusion tensor magnetic resonance imaging. Neuroimage. 2006;32(3):989-994. doi:10.1016/j.neuroimage.2006.05.044

32. Hasler R, Preti MG, Meskaldji DE, et al. Inter-hemispherical asymmetry in default-mode functional connectivity and BAIAP2 gene are associated with anger expression in ADHD adults. Psychiatry Res Neuroimaging. 2017;269:54-61. doi:10.1016/j. pscychresns.2017.09.004

33. Andrews-Hanna JR, Reidler JS, Sepulcre J, Poulin R, Buckner RL. Functional-anatomic fractionation of the brain's default network. Neuron. 2010;65(4):550-562. doi:10.1016/j.neuron.2010.02.005

34. Buckner RL, Andrews-Hanna JR, Schacter DL. The brain's default network: anatomy, function, and relevance to disease. Ann N Y Acad Sci. 2008;1124:1-38. doi:10.1196/annals.1440.011

35. Tzouriomazoyer N, Landeau B, Papathanassiou D, et al. Automated anatomical labeling of activations in SPM using a macroscopic anatomical parcellation of the MNI MRI single-subject brain. Neuroimage. 2002;15(1):273. doi:10.1006/nimg.2001.0978

36. Lowe MJ, Mock BJ, Sorenson JA. Functional connectivity in single and multislice echoplanar imaging using resting-state fluctuations. Neuroimage. 1998;7(2):119-132. doi:10.1006/nimg.1997.0315

37. Meyer M, Liem F, Hirsiger S, Jancke L, Hanggi J. Cortical surface area and cortical thickness demonstrate differential structural asymmetry in auditory-related areas of the human cortex. Cereb Cortex. 2014;24(10):2541-2552. doi:10.1093/cercor/bht094

38. Andrews-Hanna JR, Smallwood J, Spreng RN. The default network and self-generated thought: component processes, dynamic control, and clinical relevance. Ann N Y Acad Sci. 2014;1316:29-52. doi:10.1111/nyas. 12360

39. Leech R, Sharp DJ. The role of the posterior cingulate cortex in cognition and disease. Brain. 2014;137(Pt 1):12-32. doi:10.1093/ brain/awt162

40. Leech R, Braga R, Sharp DJ. Echoes of the brain within the posterior cingulate cortex. J Neurosci. 2012;32(1):215-222. doi:10.1523/ JNEUROSCI.3689-11.2012

41. Dastjerdi M, Foster BL, Nasrullah S, et al. Differential electrophysiological response during rest, self-referential, and non-selfreferential tasks in human posteromedial cortex. Proc Natl Acad Sci U S A. 2011;108(7):3023-3028. doi:10.1073/pnas.1017098108 
42. Vincent JL, Kahn I, Snyder AZ, Raichle ME, Buckner RL. Evidence for a frontoparietal control system revealed by intrinsic functional connectivity. J Neurophysiol. 2008;100(6):3328-3342. doi:10.1152/ jn. 90355.2008

43. Ko J. Neuroanatomical substrates of rodent social behavior: the medial prefrontal cortex and its projection patterns. Front Neural Circuits. 2017;11:41. doi:10.3389/fncir.2017.00041

44. Myers B, McKlveen JM, Herman JP. Glucocorticoid actions on synapses, circuits, and behavior: implications for the energetics of stress. Front Neuroendocrinol. 2014;35(2):180-196. doi:10.1016/j. yfrne.2013.12.003

45. Slavich GM, Irwin MR. From stress to inflammation and major depressive disorder: a social signal transduction theory of depression. Psychol Bull. 2014;140(3):774-815. doi:10.1037/a0035302

46. McKlveen JM, Myers B, Herman JP. The medial prefrontal cortex: coordinator of autonomic, neuroendocrine and behavioural responses to stress. J Neuroendocrinol. 2015;27(6):446-456. doi:10.1111/jne.12272

47. Johnson MK, Nolen-Hoeksema S, Mitchell KJ, Levin Y. Medial cortex activity, self-reflection and depression. Soc Cogn Affect Neurosci. 2009;4(4):313-327. doi:10.1093/scan/nsp022

48. Grimm S, Beck J, Schuepbach D, et al. Imbalance between left and right dorsolateral prefrontal cortex in major depression is linked to negative emotional judgment: an fMRI study in severe major depressive disorder. Biol Psychiatry. 2008;63(4):369-376. doi:10.1016/j. biopsych.2007.05.033

49. Chen G, Bian H, Jiang D, et al. Pseudo-continuous arterial spin labeling imaging of cerebral blood perfusion asymmetry in drug-naive patients with first-episode major depression. Biomed Rep. 2016;5(6):675-680. doi:10.3892/br.2016.796

50. Phillips ML, Drevets WC, Rauch SL, Lane R. Neurobiology of emotion perception II: implications for major psychiatric disorders. Biol Psychiatry. 2003;54(5):515-528. doi:10.1016/S0006-3223(03)00171-9

51. Cummings JL. Frontal-subcortical circuits and human behavior. Arch Neurol. 1993;50(8):873-880. doi:10.1001/archneur.1993.005400 80076020

52. Zubenko GS, Hughes HB, Hitchens TK, Cohen BM. Alterations of brain anatomy in mouse model of MDD created by replacement of homologous mouse DNA sequence with an illness-associated 6-base human CREB1 promoter sequence. Am J Med Genet B Neuropsychiatr Genet. 2014;165B (1):1-8. doi:10.1002/ajmg.b.32198

53. Lacerda AL, Brambilla P, Sassi RB, et al. Anatomical MRI study of corpus callosum in unipolar depression. J Psychiatr Res. 2005;39 (4):347-354. doi:10.1016/j.jpsychires.2004.10.004

54. Duman RS, Monteggia LM. A neurotrophic model for stress-related mood disorders. Biol Psychiatry. 2006;59(12):1116-1127. doi:10.1016/j.biopsych.2006.02.013

55. Zhang H, Li L, Wu M, et al. Brain gray matter alterations in first episodes of depression: a meta-analysis of whole-brain studies. Neurosci Biobehav Rev. 2016;60:43-50. doi:10.1016/j.neubiorev.2015.10.011
56. Poeppl TB, Muller VI, Hoffstaedter F, et al. Imbalance in subregional connectivity of the right temporoparietal junction in major depression. Hum Brain Mapp. 2016;37(8):2931-2942. doi:10.1002/ hbm. 23217

57. Grieve SM, Korgaonkar MS, Koslow SH, Gordon E, Williams LM. Widespread reductions in gray matter volume in depression. Neuroimage Clin. 2013;3:332-339. doi:10.1016/j.nicl.2013.08.016

58. Rive MM, van Rooijen G, Veltman DJ, Phillips ML, Schene AH, Ruhe HG. Neural correlates of dysfunctional emotion regulation in major depressive disorder. A systematic review of neuroimaging studies. Neurosci Biobehav Rev. 2013;37(10 Pt 2):2529-2553. doi:10.1016/j.neubiorev.2013.07.018

59. Roberts G, Wen W, Frankland A, et al. Interhemispheric white matter integrity in young people with bipolar disorder and at high genetic risk. Psychol Med. 2016;46(11):2385-2396. doi:10.1017/ S0033291716001161

60. Alexopoulos GS, Murphy CF, Gunning-Dixon FM, et al. Microstructural white matter abnormalities and remission of geriatric depression. Am J Psychiatry. 2008;165(2):238-244. doi:10.1176/ appi.ajp.2007.07050744

61. LeWinn KZ, Connolly CG, Wu J, et al. White matter correlates of adolescent depression: structural evidence for frontolimbic disconnectivity. J Am Acad Child Adolesc Psychiatry. 2014;53(8):899-909, 909 e891-897. doi:10.1016/j.jaac.2014.04.021

62. Drevets WC, Price JL, Simpson JR Jr., et al. Subgenual prefrontal cortex abnormalities in mood disorders. Nature. 1997;386 (6627):824-827. doi:10.1038/386824a0

63. Tham MW, Woon PS, Sum MY, Lee TS, Sim K. White matter abnormalities in major depression: evidence from post-mortem, neuroimaging and genetic studies. $J$ Affect Disord. 2011;132(1-2):26-36. doi:10.1016/j.jad.2010.09.013

64. Brambilla P, Nicoletti M, Sassi RB, et al. Corpus callosum signal intensity in patients with bipolar and unipolar disorder. $J$ Neurol Neurosurg Psychiatry. 2004;75(2):221-225.

65. Bartzokis G. Neuroglialpharmacology: myelination as a shared mechanism of action of psychotropic treatments. Neuropharmacology. 2012;62(7):2137-2153. doi:10.1016/j. neuropharm.2012.01.015

66. Lim JW, Donahoo AL, Bunt J, et al. EMX1 regulates NRP1-mediated wiring of the mouse anterior cingulate cortex. Development. 2015;142(21):3746-3757. doi:10.1242/dev.119909

67. Pizzagalli DA. Frontocingulate dysfunction in depression: toward biomarkers of treatment response. Neuropsychopharmacology. 2011;36(1):183-206.

68. Czeh B, Lucassen PJ. What causes the hippocampal volume decrease in depression? Are neurogenesis, glial changes and apoptosis implicated? Eur Arch Psychiatry Clin Neurosci. 2007;257 (5):250-260. doi:10.1007/s00406-007-0728-0
Neuropsychiatric Disease and Treatment

\section{Publish your work in this journal}

Neuropsychiatric Disease and Treatment is an international, peerreviewed journal of clinical therapeutics and pharmacology focusing on concise rapid reporting of clinical or pre-clinical studies on a range of neuropsychiatric and neurological disorders. This journal is indexed on PubMed Central, the 'PsycINFO' database and CAS, and is the official journal of The International Neuropsychiatric Association (INA). The manuscript management system is completely online and includes a very quick and fair peer-review system which is all easy to use. Visit http://www.dovepress.com/testimonials.php to read real quotes from published authors. 